\title{
Pressure-driven metallization in hafnium diselenide
}

Adrián Andrada-Chacón, ${ }^{* \dagger}$ Ángel Morales-García, ${ }^{\ddagger}$ Miguel A. Salvadó, ${ }^{\S}$ Pilar Pertierra, ${ }^{\S}$ Ruth Franco, ${ }^{\S}$ Gastón Garbarino, ${ }^{\|}$Mercedes Taravillo, ${ }^{\dagger}$ José A. BarredaArgüeso, Jesús González, ${ }^{\#}$ Valentín G. Baonza, ${ }^{\dagger}$ J. Manuel Recio, ${ }^{\S}$ Javier SánchezBenítez $^{\dagger}$

${ }^{\dagger}$ MALTA-Consolider Team, Departamento de Química Física, Facultad de Ciencias Químicas, Universidad Complutense de Madrid, 28040-Madrid, Spain

* Departament de Ciència de Materials i Química Física \& Institut de Química Teòrica i Computacional (IQTCUB), Universitat de Barcelona, c/ Martí i Franquès, 1-11, 08028-Barcelona, Spain

$\S$ MALTA-Consolider Team, Departamento de Química Física y Analítica, Universidad de Oviedo, 33006-Oviedo, Spain

" European Synchrotron Radiation Facility, BP 220, 6 Rue Jules Horowitz, 38043, Grenoble, Cedex 9, France

\# MALTA-Consolider Team, CITIMAC, Universidad de Cantabria, Santander, Spain

* Corresponding author. E-mail: a.andrada@ucm.es (A. Andrada-Chacón). 


\section{Supplementary Figures}

a)

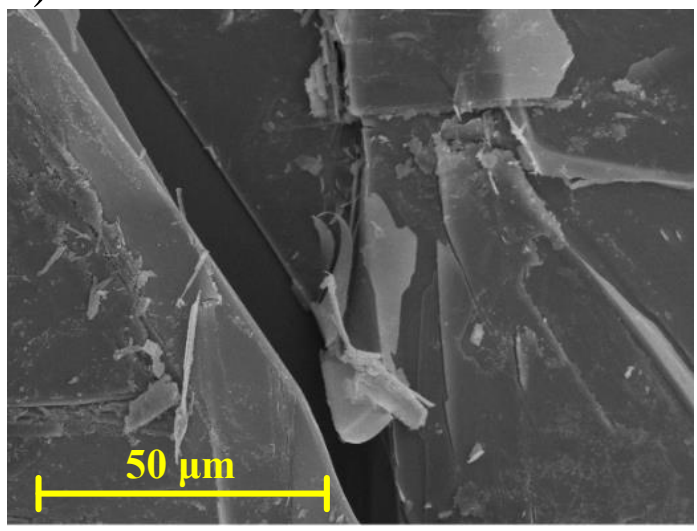

b)

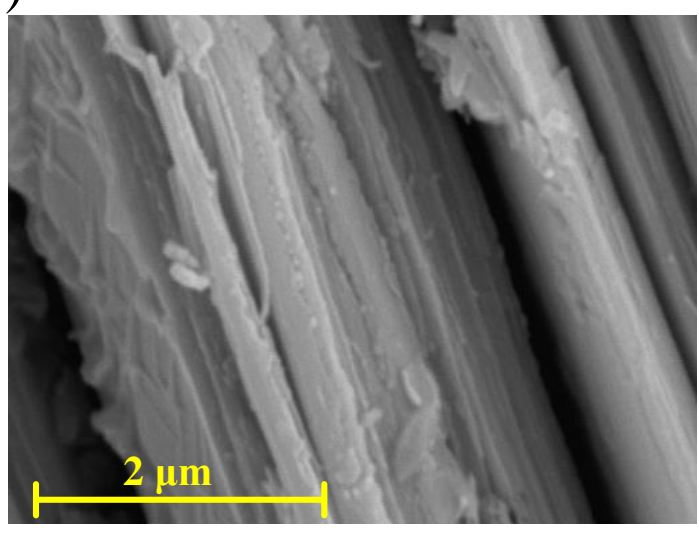

c)

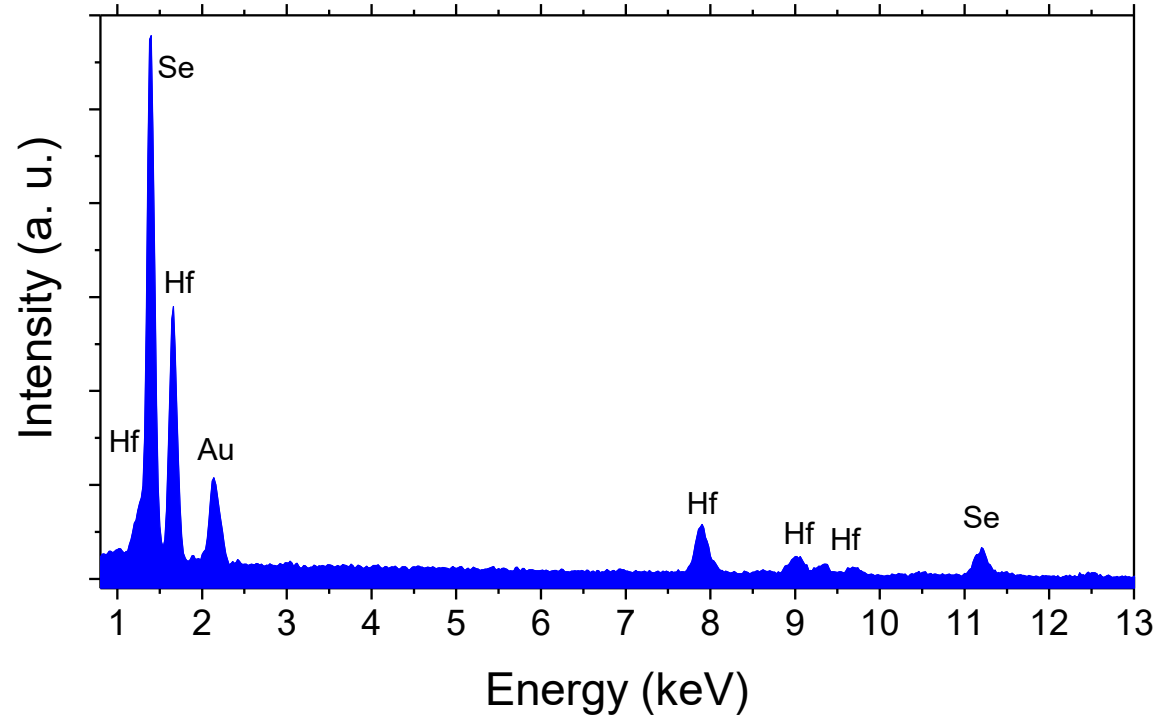

Supplementary Figure 1. Electron microscopy analysis of pristine 1T-HfSe $2 . \mathrm{SEM}$ (a) top view and (b) cross-section images of a $\mathrm{HfSe}_{2}$ flake. c) XEDS spectrum of $\mathrm{HfSe}_{2}$ crystal. 

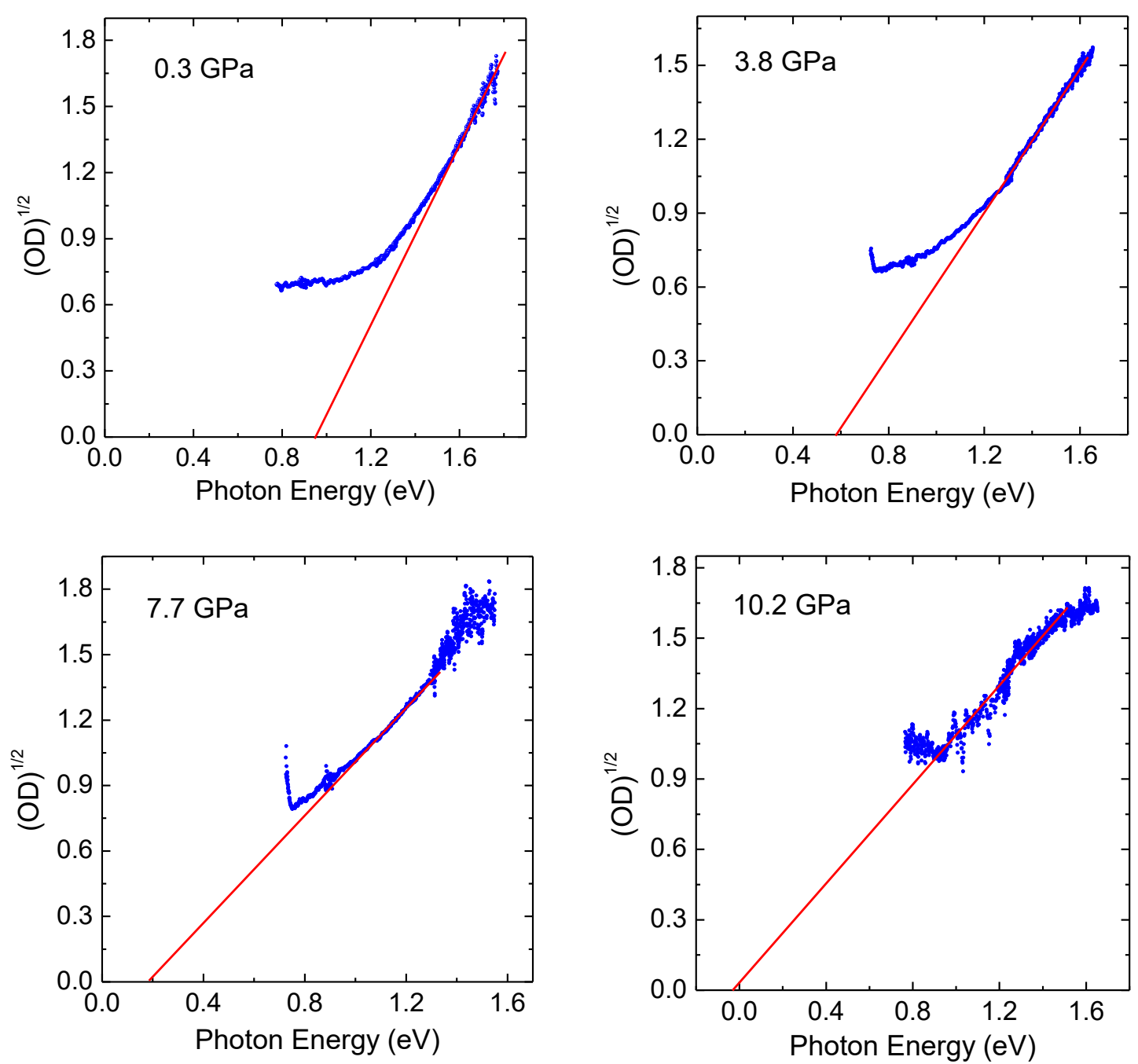

Supplementary Figure 2. Representative optical density (OD) extrapolations for the calculation of the indirect band gap at selected pressures. 


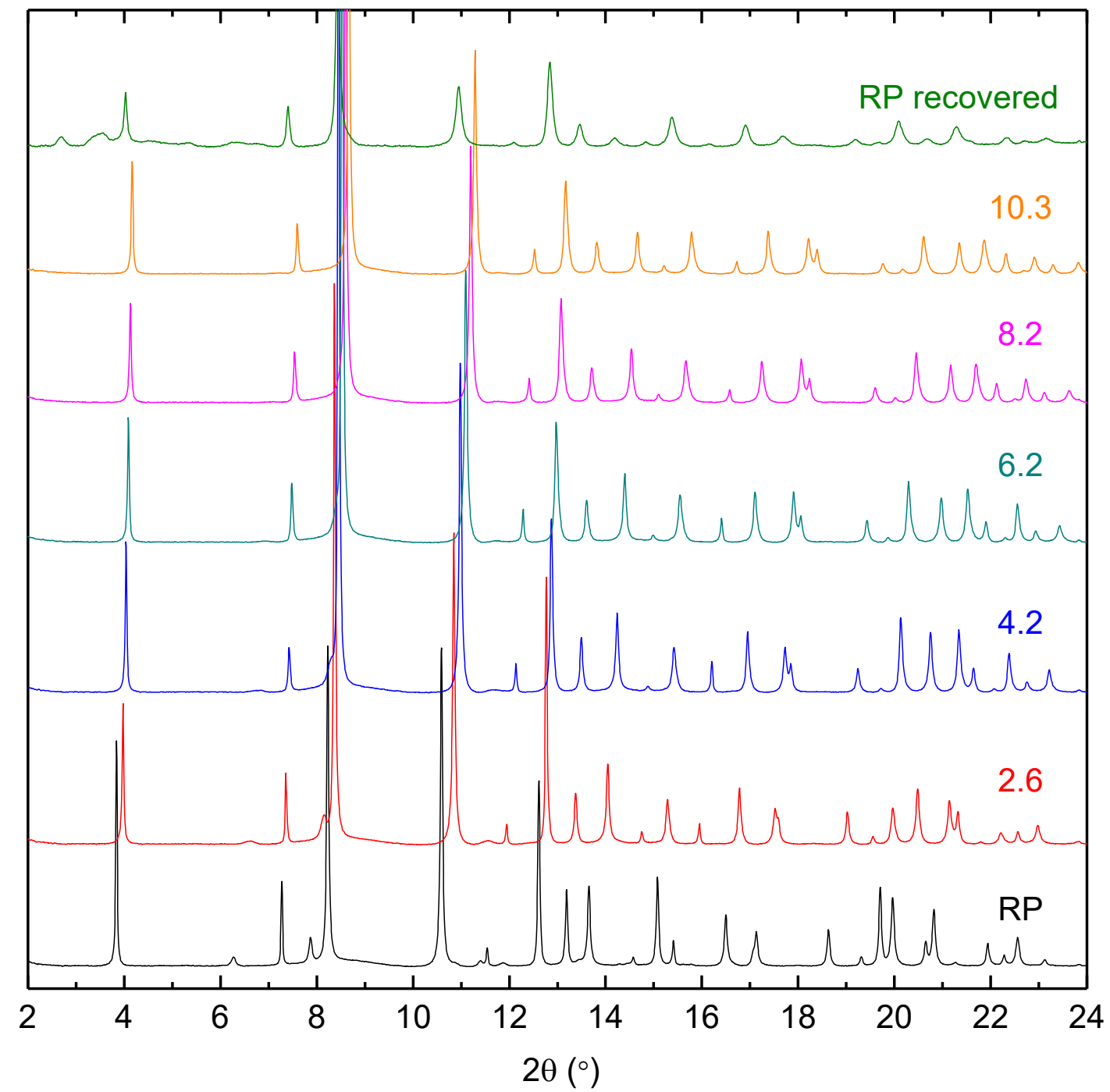

Supplementary Figure 3. Representative synchrotron XRD patterns at several pressures. Labels indicate pressures in GPa. 


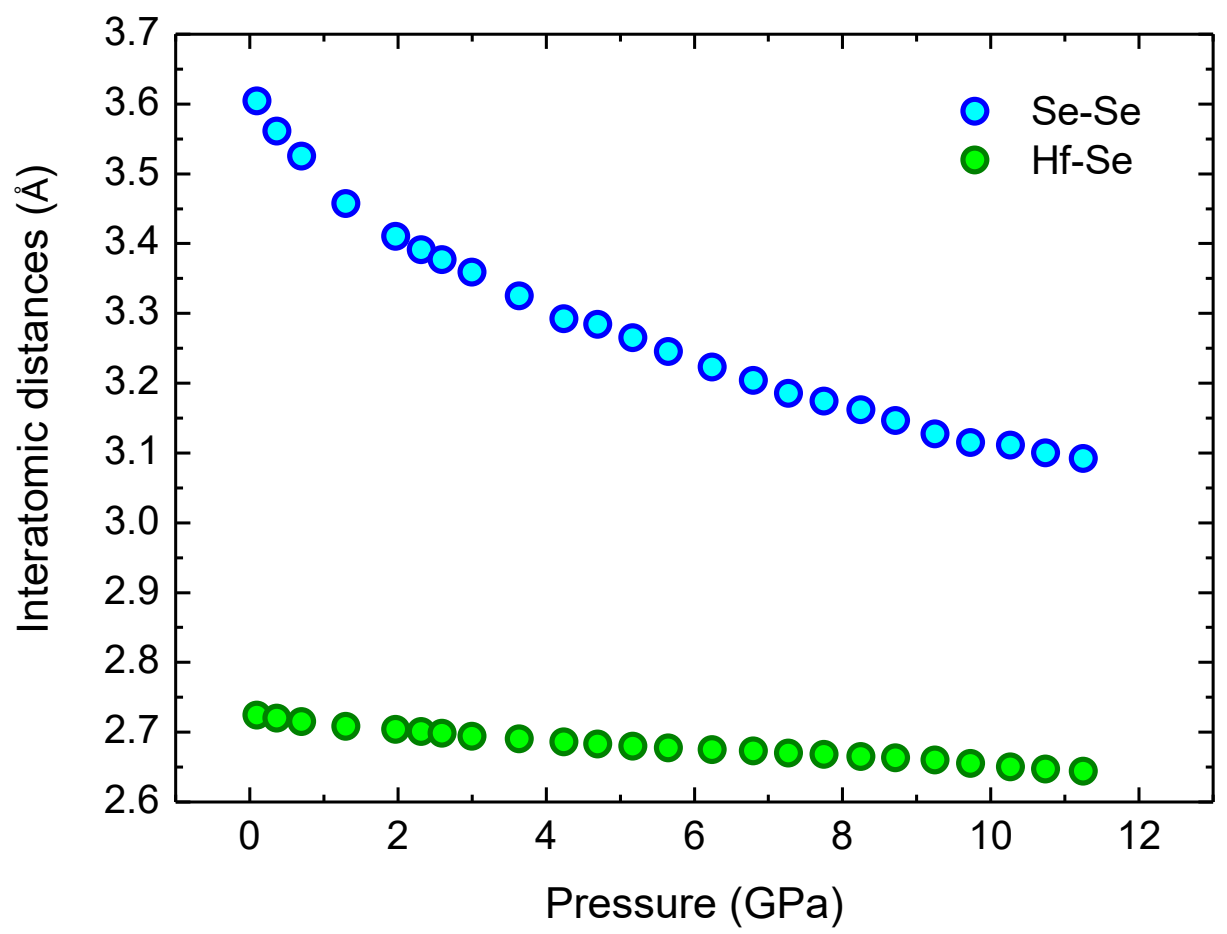

Supplementary Figure 4. Pressure evolution of Hf-Se bonding distance and Se-Se interlayer distance. 
a)

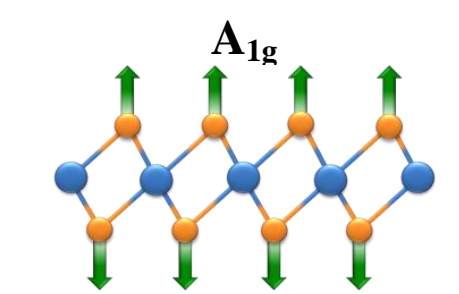

$\mathbf{A}_{2 \mathrm{u}}$

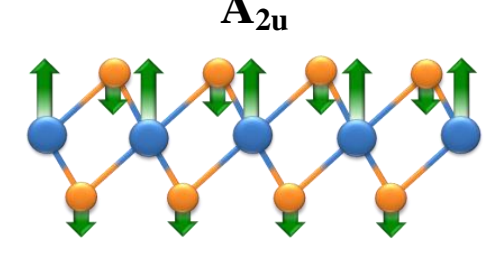

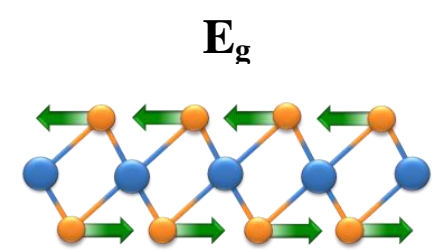

$\mathbf{E}_{\mathbf{u}}$

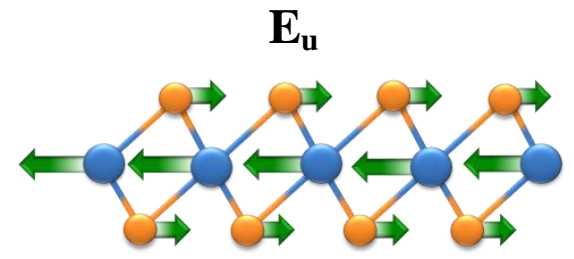

b)

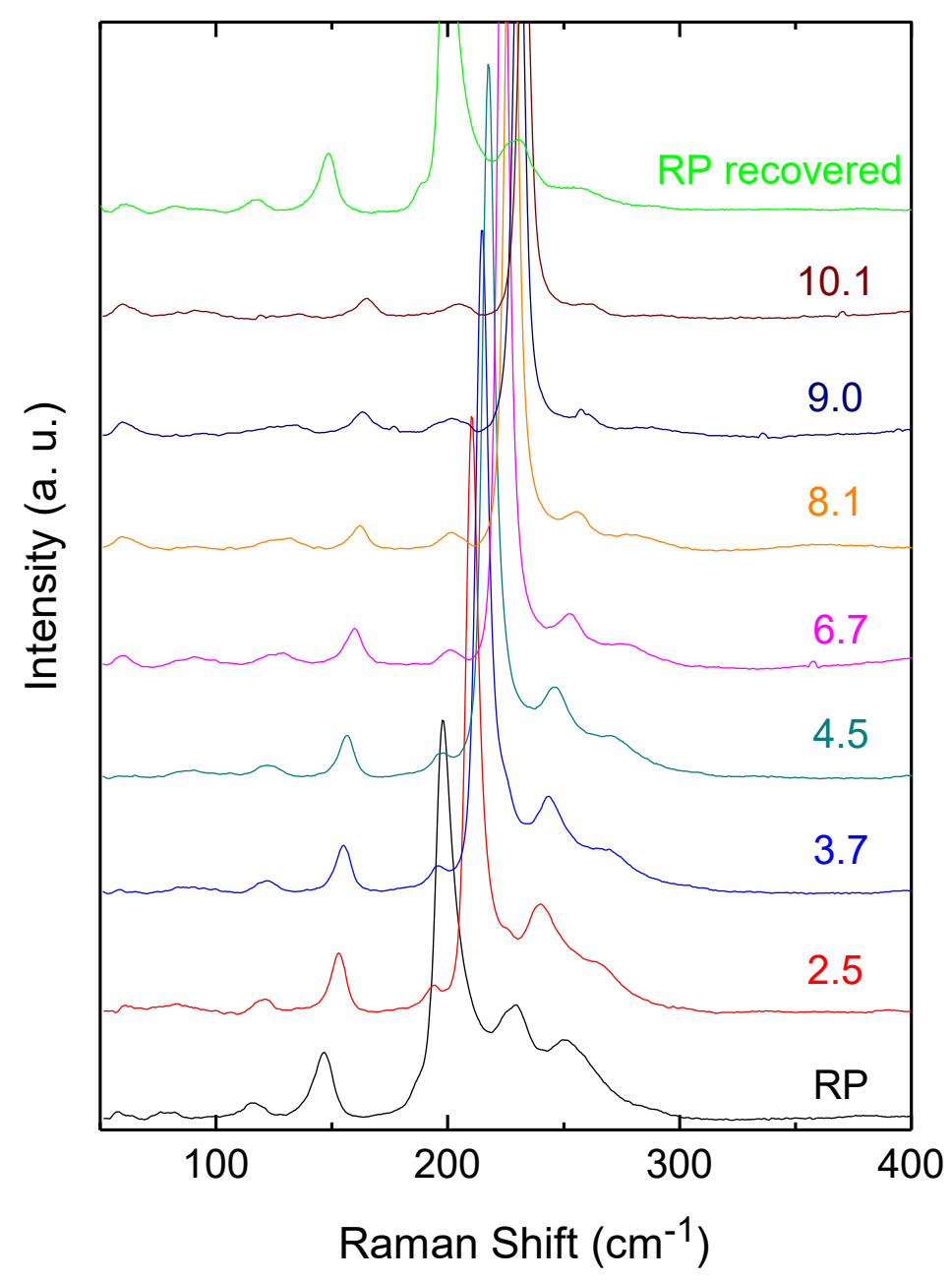

Supplementary Figure 5. a) Scheme of atomic displacements involved in optical phonons for $1 \mathrm{~T}-\mathrm{HfSe}_{2}$. Only $\mathrm{A}_{\mathrm{lg}}$ and $\mathrm{E}_{\mathrm{g}}$ phonons are active in Raman spectroscopy. $\mathrm{b}$ ) Raman spectra at several selected pressures. 


\section{Supplementary Tables}

Supplementary Table 1. Crystallographic data for $1 \mathrm{~T}-\mathrm{HfSe}_{2}$.

\begin{tabular}{|c|c|}
\hline Source & Synchrotron \\
\hline Chemical Formula & $\mathrm{HfSe}_{2}$ \\
\hline $\mathbf{T} / \mathbf{K}$ & 293 \\
\hline Pressure/bar & 1 \\
\hline Wavelength/Å & 0.4110 \\
\hline Crystal System & Trigonal \\
\hline Space Group & $P \overline{3} m 1(\# 164)$ \\
\hline$a / \AA$ & 3.7562 \\
\hline b/Å & 3.7562 \\
\hline$c / \AA$ & 6.1749 \\
\hline$\alpha / \operatorname{deg}$ & 90 \\
\hline$\beta /$ deg & 90 \\
\hline$\gamma / \operatorname{deg}$ & 120 \\
\hline $\mathrm{V} / \AA^{3}$ & 75.452 \\
\hline $\mathbf{Z}$ & 1 \\
\hline d-space range/ Å & $0.50-5.89$ \\
\hline$\chi^{2}$ & 1.54 \\
\hline $\boldsymbol{R}_{p}$ & 0.069 \\
\hline $\boldsymbol{R}_{w p}$ & 0.089 \\
\hline $\begin{array}{l}\text { Definition of } R \\
\text { factors }\end{array}$ & $\begin{array}{l}\text { Conventional definition of } R \text { factors as } \\
\text { described, for instance, in Toby (2006). }{ }^{1}\end{array}$ \\
\hline
\end{tabular}




\section{Supplementary Notes}

\section{Supplementary Note 1}

The transmittance is defined as $\mathrm{T}=\mathrm{I}_{\mathrm{T}} / \mathrm{I}_{0}$, where $\mathrm{I}_{\mathrm{T}}$ is the intensity transmitted through the sample, and $\mathrm{I}_{0}$ the light from the quartz-iodine tungsten lamp, which is measured through the pressure-transmitting medium in the cell, near the sample, by moving the DAC perpendicularly to the beam. In order to prevent errors due to light diffusion in the cell, we used a two-pinhole system. The first one is used to make a small light spot in the cell $(\varnothing \sim 30 \mu \mathrm{m})$. The second one, placed between the cell and the spectrometer, ensures that only light coming directly from the central part of the spot area is analyzed. This method reduces stray light $\left(T_{\min }\right)$ down to $8.0 \cdot 10^{-4}$. Finally, the absorption coefficient $\alpha$ is calculated from the transmittance in the absence of interference fringes, and is given by:

$$
\alpha=\frac{1}{d} \operatorname{Ln}\left[a\left(T-T_{\min }\right)\right]
$$

where $T$ is the experimental transmittance, $a$ the correction factor, and $d$ the thickness of the sample. It should be noted that the correction due to the stray light $\left(T_{\min }\right)$ has been taken into account in the high-energy, low-transmittance region.

$1 \mathrm{~T}-\mathrm{HfSe}_{2}$ is a semiconductor with an indirect band-gap. For these materials, the band gap energy, $E_{g}$, can be calculated by using the following equation: ${ }^{2,3}$

$$
\alpha(\hbar \omega) \propto\left(\hbar \omega-E_{g} \pm \hbar \Omega\right)^{2}
$$

where $\alpha(\hbar \omega)$ represents the absorption coefficient and $\hbar \omega$ is the photon energy. Plot of optical absorption coefficient square root versus photon energy (Fig. 3b inset or Fig. S2) enables to obtain the $E_{g}$ value by linear extrapolation to $\alpha=0$. Calculation of $E_{g}$ according to equation (2) introduces a very insignificant uncertainty $( \pm \hbar \Omega)$, associated to the phonon energy, which usually is much smaller than $E_{g}$. At ambient pressure, linear extrapolation yields $E_{g}$ of $1.05 \mathrm{eV}$, which is in excellent agreement with previous experimental studies for $1 \mathrm{~T}-\mathrm{HfSe}_{2}$. 


\section{Supplementary Note 2}

We have conducted several attempts and replicas of the same XRD experiment in order to obtain best quality and reliability data as possible. X-ray patterns shown in Figure S3 from ambient pressure to $10.3 \mathrm{GPa}$ correspond to our best compression stroke. Unfortunately, during decompression stroke, sample flowed out of the sample chamber in the diamond anvil cell and we were not able to acquire a recovered x-ray pattern of such sample. Other replicas allowed us to completely perform the compression/decompression cycle. Consequently, recovered x-ray pattern in Figure S3 is associated to other experimental replica. In this replica, low angle peaks $\left(2 \theta<4^{\circ}\right)$ are present from the beginning of the experiment, and they remain unaltered under pressure. In consequence, we interpret these signals as an optical artifact due to the high pressure cell or the experimental setup. In fact, according to the Rietveld analysis, sample is not predicted to exhibit Bragg positions at these $2 \theta$ values.

In addition, layered solids like $1 \mathrm{~T}-\mathrm{HfSe}_{2}$ tend to exhibit preferred orientation of the crystals. This effect is easily observed on the x-ray patterns as an anomaly in the relative intensity of certain peaks. For this reason, some differences on the relative intensity were observed when comparing the compression patterns with the recovered one. 


\section{Supplementary References}

[1] B. H. Toby. R factors in Rietveld analysis: How good is good enough? Powder Diffraction 2006, 21, $67-70$.

[2] P. Y. Yu and M. Cardona, Fundamentals of semiconductors. Physics and materials properties, Springer $4^{\text {th }}$ ed., Berlin, 2010.

[3] M. Fox, Optical properties of solids, Oxford Univ. Press, Oxford, 2010. 